\title{
Existence of Extremal Solutions of Differential Equations with Delay in Banach Spaces
}

\author{
Jinjun Fan ${ }^{1}$, Xiaodi Li ${ }^{1}$, Haydar Akca ${ }^{2 *}$ \\ 1 School of Mathematical Sciences, Shandong Normal University, Ji'nan, 250014, Shandong, China. \\ 2 Department of Mathematics, College of Arts and Science faculty, Abu Dhabi University, UAE. \\ * Corresponding author. email: Haydar.Akca@adu.ac.ae \\ Manuscript submitted February 1, 2015; accepted March 18, 2015. \\ doi: 10.17706/ijapm.2015.5.2.115-125
}

\begin{abstract}
In this paper, by using the monotone iterative method and Daher's fixed point theorem and an inequality of noncompact measure of Monch and Von Harten, we study the existence of maximum solution and minimal solution and the iterative approximation of the following initial value problem for O.D.E with delay in Banach spaces $u^{\prime}=f\left(t, u(t), u_{t}\right), u_{t 0}=\psi_{0}$ and give a sufficient condition of the existence of solutions of the above problem. The obtained results improve and generalize the corresponding results.
\end{abstract}

Key words: Banach space, measure of noncompactness, differential equation, normal cone, 2000 Mathematics Subject Classification 34G20, 47H07.

\section{Introduction}

Delay differential equations have been the important topic of research in recent years and many problems of fields of science and technology can be converted into the problems of delay differential equations. The existence of solutions of delay differential equations in a Banach space have been studied by many authors (see [1-7]). In this paper, we consider the following initial value problem of delay differential equation

$$
u^{\prime}=f\left(t, u(t), u_{t}\right), u_{t_{0}}=\psi_{0}
$$

where $f \in C\left[I \times E \times E_{0}, E\right], \psi_{0} \in E_{0}, E_{0}=C[[-\tau, 0], E], I=\left[t_{0}, t_{0}+T\right], T>0, E$ is a real Banach space with norm $\|\cdot\|, u_{t_{0}}(s)=u\left(t_{0}+s\right), s \in[-\tau, 0] \tau>0, t_{0} \in R^{+}=[0,+\infty)$.

In this paper, by using the monotone iterative method and Daher's fixed point theorem and an inequality of noncompact measure of Monch and Von Harten, we study the existence of maximum solution and minimal solution and the iterative approximation of (1), and give a sufficient condition of the existence of solutions of (1). The obtained results improve and generalize the corresponding results in [1].

\section{Preliminaries}

Let $E^{*}$ denote the dual of a real Banach space $E$ with norm $\|\cdot\|$ and $K \subset E$ be a cone, that is, a closed convex subset such that $\lambda K \subset K$ for any $\lambda \geq 0$ and $K \cap\{-K\}=\{\theta\}$. By means of $K$ a 
partial order $\leq$ is defined as $v \leq u$ iff $u-v \in K$. We let

$$
K^{*}=\left\{\phi \in E^{*}: \phi(u) \geq 0 \text { for all } u \in K\right\} .
$$

A cone $K$ is said to be normal if there exists a real number $\mu>0$ such that $0 \leq v \leq u$ implies $\|v\| \leq \mu\|u\|$, where $\mu$ is independent of $u, v$. We shall always assume in this paper that $K$ is a normal cone.

Let $\alpha, \beta$ denote the measure of noncompactness of Kuratowski and Hausdorff respectively. Their definitions are as follows

$$
\alpha(B)=\inf \left\{\varepsilon>0: B \subset U_{i=1}^{n(\varepsilon)} M_{i}^{\varepsilon} \text { for some } M_{i}^{\varepsilon} \subset E \text { with diam }\left(M_{i}^{\varepsilon}\right) \leq \varepsilon\right\}
$$

and

$$
\beta(B)=\inf \left\{\varepsilon>0: B \subset U_{i=1}^{n(\varepsilon)} S\left(x_{i}^{\varepsilon}, \varepsilon\right) \text { for some } x_{i}^{\varepsilon} \in E\right\}
$$

where $B \subset E$ bounded, $S\left(x_{i}^{\varepsilon}, \varepsilon\right)=\left\{x \in E:\left\|x-x_{i}^{\varepsilon}\right\|<\varepsilon\right\}$.

If $F$ is a subspace of $E$ and $M \subset F$ is bounded, then we define

$$
\beta_{F}(M)=\inf \left\{\varepsilon>0: M \subset \bigcup_{i=1}^{n(\varepsilon)} S\left(z_{i}^{\varepsilon}, \varepsilon\right) \text { for some } z_{i}^{\varepsilon} \in F\right\}
$$

We have

$$
\beta(B) \leq \beta_{F}(B) \leq \alpha(B) \leq 2 \cdot \beta(B) \text { for } B \subset F \text { bounded. }
$$

For these and further properties we refer to Deimling [6] and Sadovskii [8].

For any $v, w \in C[I, E]$ such that $v(t) \leq w(t)$ on $I$, we define the conical segment

$$
[v, w]=\{u \in C[I, E]: v \leq u \leq w\} .
$$

From the definition of $[v, w]$ and the normality of the cone $K$, we know that $[v, w]$ is a bounded closed convex subset of $C[I, E]$.

For any $x \in C[I, E], t \in I$, we define $x_{t}(s)=x(t+s), s \in[-\tau, 0]$. Clearly, we have $x_{t} \in E_{0}$. For any bounded set $B \subset C\left[I_{0}, E\right] \cap C^{1}[I, E]\left(I_{0}=\left[t_{0}-\tau, t_{0}+T\right]\right)$, we define

$$
B(t)=\{\phi(t): \phi \in B\}, B_{t}=\left\{\phi_{t}: \phi \in B\right\} \subset E_{0} .
$$


Let us list the following assumptions for convenience:

1) For any bounded set $B \subset C\left[I_{0}, E\right] \cap C^{1}[I, E]$,

$$
\alpha\left(f\left(I \times B(t) \times B_{t}\right)\right) \leq L \max _{s \in[-\tau, 0]} \alpha\left(B_{t}(s)\right), t \in I \text {, for some } L>0 .
$$

2) $v, w \in C\left[I_{0}, E\right] \cap C^{1}[I, E]$ with $v(t) \leq w(t)$ on $I, v_{t_{0}}(s) \leq \psi_{0}(s) \leq w_{t_{0}}(s)$ on $[-\tau, 0]$ such that $v^{\prime} \leq f\left(t, v, v_{t}\right), w^{\prime} \geq f\left(t, w, w_{t}\right)$ on $I$.

3) For any $t \in I, v(t) \leq y(t) \leq x(t) \leq w(t), v_{t}(s) \leq \varphi_{2}(s) \leq \varphi_{1}(s) \leq w_{t}(s) \quad$ on $\quad[-\tau, 0]$, $f\left(t, x(t), \varphi_{1}\right)-f\left(t, y(t), \varphi_{2}\right) \geq-M[x(t)-y(t)]-N \int_{-\tau}^{0}\left[\varphi_{1}(s)-\varphi_{2}(s)\right] d s$,

where $M, N>0$.

4) For any bounded set $B \subset C\left[I_{0}, E\right] \cap C^{1}[I, E]$,

$$
\alpha\left(f\left(t \times B(t) \times B_{t}\right)\right) \leq L \max _{s \in[-\tau, 0]} \alpha\left(B_{t}(s)\right), t \in I, \text { for some } L>0
$$

5) For any bounded set $B \subset C\left[I_{0}, E\right] \cap C^{1}[I, E]$,

$$
\alpha\left(f\left(t \times B(t) \times B_{t}\right)\right) \leq L \alpha(B), t \in I \text {, for some } L>0 \text {. }
$$

6) $v, w \in C\left[I_{0}, E\right] \cap C^{1}[I, E]$ with $v(t) \leq w(t)$ on $I$ such that there are $M>0, \quad N>0$ satisfying

$$
\begin{gathered}
\varphi\left[v^{\prime}-f\left(t, \sigma(t), \sigma_{t}\right)-M(v-\sigma)-N \int_{-\tau}^{0}\left(v_{t}(s)-\sigma_{t}(s)\right) d s\right] \leq 0, \\
\varphi\left[w^{\prime}-f\left(t, \sigma(t), \sigma_{t}\right)-M(w-\sigma)-N \int_{-\tau}^{0}\left(w_{t}(s)-\sigma_{t}(s)\right) d s\right] \geq 0
\end{gathered}
$$

for all $\sigma \in[v, w]$ and $\varphi \in K^{*}$.

In the proof of our main results the following lemmas are necessary. See [9],[10] for details.

Lemma 2.1 [9] (Daher's fixed point theorem) Let $X$ be a nonempty, bounded, closed and convex subset of a Banach space $E$. Let $A: X \rightarrow X$ be a continuous mapping such that for every countable subset $C \subset X, \alpha(A C)<\alpha(C)$ whenever $C$ is not relatively compact. Then $A$ has a fixed point.

Lemma 2.2 [10] Let $E$ be a Banach space and $\alpha$ the Kuratowski measure of noncompactness on $E$. Let $\left\{x_{n}\right\}_{n \geq 1}$ be a sequence of continuously differentiable functions from $J=[a, b]$ to $E$ such that there is some $\mu \in L^{1}(a, b)$ with $\left\|x_{n}(t)\right\| \leq \mu\left(t\right.$ and $\left\|x_{n}^{\prime}(t)\right\| \leq \mu(t)$ on $J$. Let $\varphi(t)=\alpha\left(\left\{x_{n}(t)\right\}_{n \geq 1}\right)$. Then $\varphi(t)$ is absolutely continuous on $J$ and 


$$
\varphi^{\prime}(t) \leq 2 \alpha\left(\left\{x_{n}^{\prime}(t)\right\}_{n \geq 1}\right) \text { a.e. on } J \text {. }
$$

Lemma 2.3 [10] Let $E$ be a separable Banach space and $\beta$ the Hausdorff measure of noncompactness on $E$. Let $\left\{x_{n}\right\}$ be a sequence of continuous functions from $J=[a, b]$ to $E$ such that there is some $\mu \in L^{1}(a, b)$ with $\left\|x_{n}(t)\right\| \leq \mu(t)$ on $J$. Let

$$
\phi(t)=\beta\left(\left\{x_{n}(t)\right\}_{n \geq 1}\right) .
$$

Then $\phi(t)$ is integrable on $J$ and

$$
\beta\left(\left\{\int_{a}^{b} x_{n}(s) d s\right\}_{n \geq 1}\right) \leq \int_{a}^{b} \phi(s) d s
$$

Corollary 2.1 Let $E$ be a Banach space and $\alpha$ the Kuratowski measure of noncompactness on $E$. Let $\left\{x_{n}\right\}$ be a sequence of continuously differentiable functions from $J=[a, b]$ to $E$ such that there is some $\mu \in L^{1}(a, b)$ with $\left\|x_{n}(t)\right\| \leq \mu(t)$ and $\left\|x_{n}^{\prime}(t)\right\| \leq \mu(t)$ on $J$. Let $\psi(t)=\alpha\left(\left\{x_{n}(t)\right\}_{n \geq 1}\right)$. Then $\psi(t)$ is integrable on $J$ and

$$
\alpha\left(\left\{\int_{a}^{b} x_{n}(s) d s\right\}_{n \geq 1}\right) \leq 2 \int_{a}^{b} \psi(s) d s .
$$

Proof. The absolute continuity of $\psi$ follows from

$$
\begin{aligned}
& \|\psi(t)-\psi(s)\| \leq \alpha\left(\left\{x_{n}(t)-x_{n}(s)\right\}_{n \geq 1}\right)=\alpha\left(\left\{\int_{s}^{t} x_{n}^{\prime}(\xi) d \xi\right\}_{n \geq 1}\right) \\
& \leq 2 \sup _{n \geq 1}\left|\int_{s}^{t} x_{n}^{\prime}(\xi) d \xi\right| \leq 2\left|\int_{s}^{t} \mu(\xi) d \xi\right| \quad \text { for } \quad t, s \in J .
\end{aligned}
$$

So $\psi(t)$ is integrable on $J$.

Let

$$
S=\left\{x_{n}(t): n \geq 1 \text { and } t \in J \cap Q\right\} \text { and } F=\overline{\operatorname{span} S}
$$

where $Q$ is a rational number set. Then $F$ is a separable closed subspace of $E$ and

$$
\left\{\int_{a}^{b} x_{n}(s) d s: n \geq 1\right\} \subset F,\left\{x_{n}(t): n \geq 1, t \in[a, b]\right\} \subset F .
$$

By the properties of $\alpha$ and Lemma 2.3, we have 


$$
\begin{aligned}
& \alpha\left(\left\{\int_{a}^{b} x_{n}(s) d s\right\}_{n \geq 1}\right) \leq 2 \beta_{F}\left(\left\{\int_{a}^{b} x_{n}(s) d s\right\}_{n \geq 1}\right) \\
& \leq 2 \int_{a}^{b} \beta_{F}\left(\left\{x_{n}(s)\right\}_{n \geq 1}\right) d s \leq 2 \int_{a}^{b} \alpha\left(\left\{x_{n}(s)\right\}_{n \geq 1}\right) d s=2 \int_{a}^{b} \psi(s) d s .
\end{aligned}
$$

The proof is complete.

\section{Main Results}

In [1] S. W. Du established the following

Theorem 3.1 Assume that the cone $K$ is normal and assumptions $\left(A_{1}\right),\left(A_{2}\right)$ and $\left(A_{3}\right)$ are satisfied. Then there exist two sequences $\left\{v_{n}\right\},\left\{w_{n}\right\}$ on $I$ such that

$$
v \leq v_{1} \leq v_{2} \leq \cdots \leq v_{n} \leq w_{n} \leq \cdots \leq w_{2} \leq w_{1} \leq w
$$

and

$$
\lim _{n \rightarrow+\infty} v_{n}(t)=\rho(t), \lim _{n \rightarrow+\infty} w_{n}(t)=\gamma(t)
$$

uniformly on $I$, where $\rho, \gamma$ are minimal and maximal solutions of (1).

Our main aim is to prove the following Theorem 3.2 and Theorem 3.3.

Theorem 3.2 Assume that the cone $K$ is normal and assumptions $\left(A_{2}\right),\left(A_{3}\right)$ and $\left(A_{4}\right)$ are satisfied. Then there exist two sequences $\left\{v_{n}\right\},\left\{w_{n}\right\}$ on $I$ such that

$$
v \leq v_{1} \leq v_{2} \leq \cdots \leq v_{n} \leq w_{n} \leq \cdots \leq w_{2} \leq w_{1} \leq w
$$

and

$$
\lim _{n \rightarrow+\infty} v_{n}(t)=\rho(t), \lim _{n \rightarrow+\infty} w_{n}(t)=\gamma(t)
$$

uniformly on $I$, where $\rho, \gamma$ are minimal and maximal solutions of (1)

Note. Obviously, the condition of $\left(A_{4}\right)$ is weaker than that of $\left(A_{1}\right)$. The result of Theorem 3.2 is the extension of that of Theorem 3.1.

Theorem 3.3 Assume that the cone $K$ is normal and assumptions $\left(A_{5}\right)$ and $\left(A_{6}\right)$ are satisfied. Then there exists a solution $u(t)$ of $(1.1)$ such that $v(t) \leq u(t) \leq w(t)$ on $I$.

\section{The Proof of Theorem 3.2}

We first, as in [1], consider the linear differential equation with delay

$$
u^{\prime}=F\left(t, u(t), u_{t}\right), u_{t_{0}}=\psi_{0}
$$


where $F\left(t, u(t), u_{t}\right)=f\left(t, \eta(t), \eta_{t}\right)-M[u(t)-\eta(t)]-N \int_{-\tau}^{0}\left[u_{t}(s)-\eta_{t}(s)\right] d s$ and $\eta \in[v, w]$.

As the proof of Lemma 3.1 in [1], (2) has an unique solution $u(t)$. Thus we define the mapping $A$ by $A \eta=u$.

Concerning the mapping $A$, the following properties is proved in [1].

1) $v \leq A v, w \geq A w ; \quad A([v, w]) \subset[v, w]$;

2) $A \eta_{1} \leq A \eta_{2}$ if $\eta_{1}, \eta_{2} \in[v, w], \eta_{1} \leq \eta_{2}$ and $\eta_{1, t_{0}}=\eta_{2, t_{0}}=\psi_{0}$.

We define two sequences $\left\{v_{n}\right\},\left\{w_{n}\right\}$ by

$$
v_{n}=A v_{n-1}, w_{n}=A w_{n-1}(n=1,2, \cdots)
$$

with $v_{0}=v, w_{0}=w$. Obviously,

$$
v=v_{0} \leq v_{1} \leq v_{2} \leq \cdots \leq v_{n} \leq w_{n} \leq \cdots \leq w_{2} \leq w_{1} \leq w_{0}=w
$$

Thus $\left\{v_{n}\right\}_{n \geq 0},\left\{w_{n}\right\}_{n \geq 0}$ are uniformly bounded on $I$. By (2) and $\left(A_{4}\right),\left\{v_{n}^{\prime}\right\}_{n \geq 1},\left\{w_{n}^{\prime}\right\}_{n \geq 1}$ are uniformly bounded on $I$, too. Therefore, $\left\{v_{n}\right\}_{n \geq 0}\left\{w_{n}\right\}_{n \geq 0}$ are equicontinuous.

Let

$$
Z(t)=\left\{v_{n}(t)\right\}_{n \geq 0}, Z_{t}(s)=\left\{v_{n, t}(s)\right\}_{n \geq 0}, m(t)=\alpha(Z(t)), m_{t}(s)=\alpha\left(Z_{t}(s)\right), t \in I, s \in[-\tau, 0] .
$$

Obviously the sequence $\left\{v_{n}\right\}_{n \geq 0}$ satisfies the conditions of Lemma 2.2 and Corollary 2.1. Using the properties of measure of noncompactness and the condition $\left(A_{4}\right)$, we have, by Lemma 2.2 and Corollary 2.1,

$$
\begin{gathered}
D^{+} m(t) \leq 2 \alpha\left(\left\{v_{n}^{\prime}(t)\right\}_{n \geq 1}\right) \\
=2 \alpha\left(\left\{f\left(t, v_{n-1}(t), v_{n-1, t}\right)-M\left[v_{n}(t)-v_{n-1}(t)\right]-N \int_{-\tau}^{0}\left[v_{n, t}(s)-v_{n-1, t}(s)\right] d s\right\}_{n \geq 1}\right) \\
\leq 2 \alpha\left(\left\{f\left(t, v_{n-1}(t), v_{n-1, t}\right)\right\}_{n \geq 1}\right)+4 M m(t)+2 N \alpha\left(\left\{\int_{-\tau}^{0}\left(v_{n, t}(s)-v_{n-1, t}(s)\right) d s\right\}_{n \geq 1}\right) \\
\leq 2 \alpha\left(f\left(t, Z(t), Z_{t}\right)\right)+4 M m(t)+8 N \int_{-\tau}^{0} \alpha\left(Z_{t}(s)\right) d s \\
\leq 2 L \max _{s \in[-\tau, 0]} m_{t}(s)+4 M m(t)+8 N \tau \max _{s \in[-\tau, 0]} m_{t}(s) \\
\leq(2 L+4 M+8 N \tau) \max _{s \in[-\tau, 0]} m_{t}(s) .
\end{gathered}
$$

Noting $m_{t_{0}}=\alpha\left(\left\{\psi_{0}\right\}\right)=0$, the above estimation yields $m(t) \equiv 0$ on $I$ by a standard result (Theorem 1.6.6 in [11]). So $\left\{v_{n}(t)\right\}_{n \geq 0}$ is relatively compact for each $t \in I$. Similarly, $\left\{w_{n}(t)\right\}_{n \geq 0}$ is 
relatively compact for each $t \in I$. Therefore, by the Ascoli-Arzela theorem, the sequences $\left\{v_{n}(t)\right\}_{n \geq 0}$ and $\left\{w_{n}(t)\right\}_{n \geq 0}$ have uniformly convergent subsequences. This, together with the monotone character of each one of these sequences, implies that full sequences converge uniformly on $I$. Let $\lim _{n \rightarrow+\infty} v_{n}(t)=\rho(t), \lim _{n \rightarrow+\infty} w_{n}(t)=\gamma(t)$, where the convergence is uniform on $I$. By (2), $\rho(t), \gamma(t)$ are solutions of (1). Moreover, if $u(t)$ is a solution of (1) such that $u \in[v, w]$, then by using induction arguments, we can show that $v_{n} \leq u \leq w_{n}$ holds for all $n$. This implies that $v \leq u \leq w$ on $I$, which means that $\rho, \gamma$ are indeed minimal and maximal solutions of (1) and the proof is complete.

\section{The Proof of Theorem 3.3}

The proof of Theorem 3.3 will be completed by two steps.

Step 1. Assume that the number $T$ is small enough such that

$$
0<T<\frac{1}{2(L+2 M+4 N \gamma)}
$$

we first consider the linear IVP of differential equations with delay

$$
u^{\prime}=F_{1}\left(t, u(t), u_{t}\right), u_{t_{0}}=\psi_{0}
$$

where

$$
F_{1}\left(t, u(t), u_{t}\right)=f\left(t, \eta(t), \eta_{t}\right)+M[u(t)-\eta(t)]+N \int_{-\tau}^{0}\left[u_{t}(s)-\eta_{t}(s)\right] d s
$$

and $\eta \in[v, w]$.

As the proof of Lemma 3.1 in [1], (3) has an unique solution $u$. Thus we define the mapping $A$ by $A \eta=u$.

Concerning the mapping $A$, there are the following properties.

1) $A$ maps the sector $[v, w]$ into itself.

2) $A:[v, w] \rightarrow[v, w]$ is continuous.

In fact, for any $\eta \in[v, w]$ and $\phi \in K^{*}$, we let

$$
A \eta=u, P(t)=\varphi(v(t)-u(t)), P_{t}(s)=\varphi\left(v_{t}(s)-u_{t}(s)\right), t \in I, s \in[-\tau, 0],
$$

Then

$$
\begin{gathered}
D^{+} P(t)=\varphi\left(v^{\prime}(t)-u^{\prime}(t)\right) \\
\leq \phi\left(f\left(t, \sigma(t), \sigma_{t}\right)+M(v(t)-\sigma(t))+N \int_{-\tau}^{0}\left[v_{t}(s)-\sigma_{t}(s)\right] d s\right. \\
\left.-f\left(t, \eta(t), \eta_{t}\right)-M(u(t)-\eta(t))-N \int_{-\tau}^{0}\left[u_{t}(s)-\eta_{t}(s)\right] d s\right)
\end{gathered}
$$


Using the assumption $\left(A_{6}\right)$ with $\sigma=\eta$, the above inequality implies

$$
\begin{aligned}
D^{+} P(t) \leq \phi( & \left.M(v(t)-u(t))+N \int_{-\tau}^{0}\left[v_{t}(s)-u_{t}(s)\right] d s\right) \\
& =M P(t)+N \int_{-\tau}^{0} P_{t}(s) d s \\
\leq & (M+N \tau) \max _{s \in[-\tau, 0]} P_{t}(s)
\end{aligned}
$$

Noting $P_{t_{0}} \leq 0$, as the proof of Lemma 3.2 in [1], we have $P(t) \leq 0$ on $I$. Since $\phi \in K^{*}$ is arbitrary, we have $v(t) \leq u(t)$ on $I$. Similarly, we can obtain $u(t) \leq w(t)$ on $I$. Therefore $A[v, w] \subset[v, w]$, i.e. (i) holds.

To prove (ii), let $u_{n} \in[v, w], \lim _{n \rightarrow \infty} u_{n}=u$. Obviously $u \in[v, w]$. Because the interval $I=\left[t_{0}, t_{0}+T\right]$ is compact and $f$ is continuous, it is easy to see

$$
\begin{gathered}
\left\|f\left(t, u_{n}(t), u_{n, t}\right)-f\left(t, u(t), u_{t}\right)\right\| \rightarrow 0 \text { as } n \rightarrow \infty, \\
\left\|f\left(t, u_{n}(t), u_{n, t}\right)-f\left(t, u_{m}(t), u_{m, t}\right)\right\| \rightarrow 0 \text { as } m, n \rightarrow \infty
\end{gathered}
$$

uniformly on $I$.

By (5) and

$$
\begin{gathered}
A u_{n}(t)-A u_{m}(t)=\int_{t_{0}}^{t}\left\{\left[f\left(s, u_{n}(s), u_{n . s}\right)-f\left(s, u_{m}(s), u_{m, s}\right)\right]\right\} d s \\
+\int_{t_{0}}^{t}\left\{M\left[A u_{n}(s)-A u_{m}(s)\right]+M\left[u_{m}(s)-u_{n}(s)\right]\right\} d s \\
+N \int_{t_{0}}^{t}\left\{\int_{-\tau}^{0}\left[A u_{n, s}(\xi)-A u_{m, s}(\xi)\right] d \xi+\int_{-\tau}^{0}\left[u_{m, s}(\xi)-u_{n, s}(\xi)\right] d \xi\right\} d s,
\end{gathered}
$$

we have

$$
\left\|A u_{n}-A u_{m}\right\| \leq \frac{T}{1-(M+N \tau) T}\left[\max _{t \in I}\left\|f\left(t, u_{n}(t), u_{n, t}\right)-f\left(t, u_{m}(t), u_{m, t}\right)\right\|+(M+N \tau)\left\|u_{n}-u_{m}\right\|\right] .
$$

Hence

$$
\left\|A u_{n}-A u_{m}\right\| \rightarrow 0 \text { as } n, m \rightarrow \infty
$$

Since $E$ is a Banach space, there exists a $\bar{u}$ such that $\lim _{n \rightarrow \infty} A u_{n}=\bar{u}$. It is easy to see, from the definition of $A$ and (4), that 


$$
\lim _{n \rightarrow \infty} A u_{n}=\bar{u}=A u
$$

Therefore $A:[v, w] \rightarrow[v, w]$ is continuous.

Now we will verify that the conditions of Lemma 2.1 are satisfied. For any countable subset $C \subset[v, w]$ with $\alpha(C)>0$, we let $\psi(t)=\alpha\left(\left\{A u_{n}(t): u_{n} \in C\right\}\right)$. Obviously the sequence $\left\{A u_{n}(t)\right\}_{n \geq 1}$ satisfies the conditions of Lemma 2.2. Using the properties of measure of noncompactness, we have, by Lemma 2.2 and Corollary 2.1,

$$
\begin{gathered}
\psi^{\prime}(t) \leq 2 \alpha\left(\left\{\left(A u_{n}(t)\right)^{\prime}: u_{n} \in C\right\}\right) \\
=2 \alpha\left(\left\{f\left(t, u_{n}(t), u_{n, t}\right)+M\left(A u_{n}(t)-u_{n}(t)\right)+N \int_{-\tau}^{0}\left[A u_{n, t}(s)-u_{n, t}(s)\right] d s: u_{n} \in C\right\}\right) \\
\leq 2 L \alpha(C)+2 M \alpha(C)+2 M \alpha(A C)+4 N \tau \alpha(A C)+4 N \tau \alpha(C) \\
=(2 M+4 N \tau) \alpha(A C)+(2 L+2 M+4 N \tau) \alpha(C) \text { a.e. on } I .
\end{gathered}
$$

Thus, integrating this from $t_{0}$ to $t, t \in\left[t_{0}, t_{0}+T\right]$, and rearranging, we have

$$
\alpha(A C) \leq \frac{T(2 L+2 M+4 N \tau)}{1-(2 M+4 N \tau) T} \alpha(C)<\alpha(C)
$$

By Lemma 2.1, we conclude that there exists a $u \in[v, w]$ such that $A u=u$ and $u$ is a solution of (1) on $I$.

Step 2. If $T$ is not sufficiently small, we divide the interval $\left[t_{0}, t_{0}+T\right]$ into $k$ equal subintervals $t_{0}<t_{1}<\cdots<t_{k}=t_{0}+T$ such that the length $T_{0}=t_{i+1}-t_{i}$ of each subinterval meets the needs of step 1 , i.e., $0<T_{0}<\frac{1}{2(L+2 M+4 N \tau)}$. By step 1 , there exists a solution $u_{1}(t)$ of $(1)$ defined on $\left[t_{0}-\tau, t_{1}\right]$. We consider the following IVP

$$
\left\{\begin{array}{l}
u^{\prime}=f\left(t, u(t), u_{t}\right), t \in\left[t_{1}, t_{2}\right] \\
u_{t_{1}}(s)=u_{1}\left(t_{1}+s\right), s \in[-\tau, 0] .
\end{array}\right.
$$

By step 1 , there exists a solution $u_{2}$ of $(I V P)_{1}$ defined on $\left[t_{1}-\tau, t_{2}\right]$. Similarly, we consider the following IVP

$$
\left\{\begin{array}{l}
u^{\prime}=f\left(t, u(t), u_{t}\right), t \in\left[t_{i}, t_{i+1}\right] \\
u_{t_{i}}(s)=u_{i}\left(t_{i}+s\right), s \in[-\tau, 0]
\end{array}\right.
$$


By step 1 , there exists a solution $u_{i+1}$ of $(I V P)_{i}$ defined on $\left[t_{i}-\tau, t_{i+1}\right]$. It is easy to verify that

$$
u(t)=u_{i}(t), t \in\left[t_{i-1}, t_{i}\right](i=1,2, \cdots, k)
$$

is a solution of (1) on $I$ such that $u \in[v, w]$. The proof is complete.

\section{References}

[1] Du, S. W. (1983). Monotone Iterative Technique for Delay Differential Equations in Abstract Cones. New York: Elsevier Science Publishing Co. Inc.

[2] Lakshmikantham, V., Leela, S., \& Moauro, V. (1978). Existence and uniqueness of solutions of delay differential equations on a closed subset of a banach space. Nonlinear Analysis, 2, 311-327,

[3] Leela, S., \& Moauro, V. (1978). Existence of solutions of delay differential equations on a closed subset of a banach space. Nonlinear Analysis, 2, 47-58,

[4] Kato, S. (1995). Existence, uniqueness and continuous dependence of solutions of delay differential equations with infinite delays in a banach space. J. Math. Anal. Appl., 1, 82-91,

[5] Hal, S. (2011). An introduction to delay differential equations with applications to life sciences. Texts in Applied Mathematics. London, New York: Springer.

[6] Deimling, K. (1977). Ordinary Differential Equations in Banach Spaces. Berlin-Heidelberg, New York.

[7] Fu, X.-L., \& Fan, J.-J. (2011). Nonlinear Differential Equations. Beijing: Scientific Publishing House.

[8] Sadovskii, B. N. (1972). Limit-compact and condensing operators. Russian Math. Surveys, 27, 85-155,

[9] Daher, S. J. (1978). On a fixed point principle of sadovski. Nonlinear Anal., 2, 643-645,

[10] Monch, H., \& Harten, V. (1982). On the cauchy problem for O.D.E in banach spaces. Arch. Math, 39, 153-160.

[11] Lakshmikantham, V., \& Leela, S. (1981). Nonlinear Differential Equations in Abstract Spaces. Oxford: Pergamon.

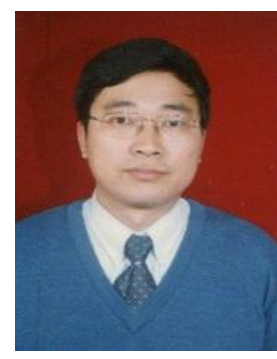

Jinjun Fan was born in Shandong province, China. He received the B.S. and M.S. degrees from Shandong Normal University, Jinan, China, in 1985 and 1988, respectively, all in applied mathematics. He is currently a professor with the Department of Mathematics, Shandong Normal University. He has authored or coauthored more than 40 research papers. His current research interests include differential equations in banach spaces, boundary value problems, discontinuous dynamical systems and differential equations on time scales.

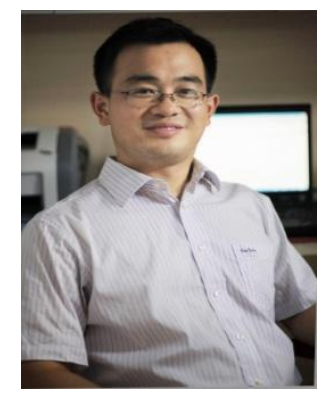

Xiaodi Li was born in Shandong province, China. He received the B.S. and M.S. degrees from Shandong Normal University, Jinan, China, in 2005 and 2008, respectively, and the Ph.D. degree from Xiamen University, Xiamen, China, in 2011, all in applied mathematics. He is currently a professor with the Department of Mathematics, Shandong Normal University. He has authored or coauthored more than 50 research papers. His current research interests include stability theory, artificial neural networks, impulsive control systems and applied mathematics. 
Haydar Akca graduated in mathematics and astronomy from Ege University, Faculty of Science, in 1970. Dr. Akca received his Ph. D. in applied mathematics from Inonu University, Malatya with collaboration Helsinki University of Technology in 1983. Since then, he has been teaching in various universities. He became a professor in applied mathematics at Akdeniz University, Antalya in 1996. He has around 95 technical publications including two monographs, and his research interest area includes primarily functional differential equations, neural networks, mathematical modeling, control theory, and wavelet neural networks. He has been organizing the serial international conference on dynamical systems and applications. Dr. Akca is a member of a number of professional mathematical associations. He is the editor-in-chief and editorial board member of number of international mathematical journals. At present he is professor of applied mathematics at the Abu Dhabi University, College of Arts and Science Department of Applied Sciences and Mathematics, Abu Dhabi, United Arab Emirates. 